\title{
Does the Addition of Visual Feedback Improve Postural Vertical Training in the Patients with Pusher Syndrome After Stroke?
} Jang-Tae Lee, PT, BSc · Seung-Chul Chon, PT, PhD ${ }^{1 \dagger}$

Dept. of Physical Therapy, Graduate School, Konyang University

${ }^{1}$ Dept. of Physical Therapy, College of Medical Science, Konyang University

Received: July 5, 2017 / Revised: July 13, 2017 / Accepted: July 30, 2017

(C) 2017 J Korean Soc Phys Med

\section{| Abstract |}

PURPOSE: To compare postural vertical training with and without visual feedback for improving functional recovery in post-stroke hemiparesis patients with pusher syndrome.

METHODS: This study used a single-subject research with alternating design with multiple baselines. Three patients with hemiparetic post-stroke diagnosed with pusher syndrome were selected from the inpatients at the department of physical therapy of a local rehabilitation hospital. For subjective postural vertical (SPV) training with and without visual feedback, an alternating treatment was used. The subjects were randomly selected using the sequence of the two training methods upon starting the intervention, and then the training was alternated. SPV training was performed twice a day, once in the morning and again in the afternoon. Scale for contraversive pushing (SCP), postural assessment scale for stroke, and Barthel index score were used to determine the intervention-related changes.

$\dagger$ Corresponding Author : keyjune@konyang.ac.kr This is an Open Access article distributed under the terms of the Creative Commons Attribution Non-Commercial License (http://creativecommons.org/licenses/by-nc/3.0) which permits unrestricted non-commercial use, distribution, and reproduction in any medium, provided the original work is properly cited.
RESULTS: Compared to the average score at baseline, the average SCP score for the SPV training without visual feedback decreased from 5.3 to 2.8 , from 4.6 to 3 , and from 3.5 to 2.7 for subjects 1,2 , and 3 , respectively. However, the average score for the SPV training with visual feedback decreased from 5.3 to 3.1 , from 4.6 to 3.5 , and from 3.5 to 3.3 for subjects 1,2 , and 3 , respectively.

CONCLUSION: Postural vertical training without visual feedback may be more beneficial than postural vertical training with visual feedback for improving pushing behavior and functional activity in stroke patients with pusher syndrome.

Key Words: Postural vertical training, Pusher syndrome, Rehabilitation, Stroke, Visual feedback

\section{Introduction}

Pusher syndrome is when post-stroke patients push toward the affected side of their body, which is contrary to the usual behavior post-stroke. This syndrome results in slow recovery, and the long-term period is a negative prognostic factor, despite good levels of muscle strength and motor function (Karnath and Broetz, 2003). To improve this syndrome, visual feedback has been used in those with 
pusher syndrome based on normal subjective visual vertical and abnormal subjective postural vertical concepts (Karnath and Broetz, 2003).

The subjective visual vertical concept is described by a person sitting in a chair to represent the slope of the straight line in order to see the fluorescent light projected on the wall in a dark room, which pusher syndrome patients show a normal response to. Conversely, the subjective postural vertical concept is measured by the vertical orientation of the perceived body. After irregularly rotating the chair, subjects sitting in the chair in the dark represent the vertical position, and it has been reported that subjects incorrectly recognized the approximately $20^{\circ}$ of tilt on the unaffected side (Bonan et al., 2007; Karnath and Broetz, 2003). Based on these two tests, visual feedback has been provided to control this abnormal subjective postural vertical for functional recovery, and the effect of visual feedback provided to those with pusher syndrome has been recognized as an effective treatment (Broetz et al., 2004; Karnath et al., 2000).

However, the proprioceptive sensory factor, which is the most important in balance and postural control, was excluded from the experimental conditions in the subjective postural vertical and subjective visual vertical tests and was only used in visual and vestibular sensory systems (Karnath et al., 2000; Karnath and Broetz, 2003). For example, to control standing balance in stroke patients, the proprioceptive sense of the soles of their feet was reported as the most important factor (Priplata et al., 2006).

The subjective postural vertical training blocks visual feedback of the vertical orientation, which is beneficial for providing sufficient time for the proprioceptive sensory to be more immersed and recruited for balance control. It is an inaccurate integration of visual and cognitive feedback, and since the central nervous system can be used selectively, visual feedback was not necessary to control posture and balance (Shumway-Cook and Woollacott., 1995). In addition, mirrors usually provide visual feedback, showing the left and right reversed, which may be confusing to the patient during the adjustment of sensory organization (Di Fabio and Badke, 1990). Consequently, for sensory re-education of the affected side, the same side should be shown in the mirror as visual feedback, not the reversed side (Stevens and Stoykov, 2003).

The concepts of normal subjective visual vertical and abnormal subjective postural vertical have been reported on (Karnath and Broetz, 2003); however, in the clinical rehabilitation field, the subjective postural vertical concept of blocking visual feedback has been used as an unvarying way and is associated with specific clinical research that is still lacking. Thus, this present study aimed to compare the effect of subjective postural vertical training with visual feedback and without visual feedback on the functional motor recovery including the degree of pushing and balance in post-stroke hemiparesis patients with pusher syndrome.

\section{Methods}

\section{Subjects}

Three patients with hemiparetic post-stroke diagnosed with pusher syndrome were selected from the inpatients at the department of physical therapy of a local rehabilitation hospital. The selection criteria were as follows: 1) no significant cognitive deficits of $>25$ points on the Mini-Mental Status Examination, 2) no apraxia, 3) $>2$ months after the onset of stroke, and 4) an average score of $>5$ on the Scale for Contraversive Pushing (SCP) for those with serious pusher syndrome. All subjects received routine physical therapy, which included simple range of motion exercises, stretching, and massage during the study, none of which were related to the pushing behaviors. The general characteristics of the subjects including sex, age, weight, height, modified Ashworth scale (MAS), Mini-Mental Status Examination (MMSE), speech, duration, and SCP measurement are presented in Table 1. 
Table 1. Subjects' characteristics

\begin{tabular}{cccccccccc}
\hline Subject & Sex & $\begin{array}{c}\text { Age } \\
(\text { years })\end{array}$ & $\begin{array}{c}\text { Weight } \\
(\mathrm{kg})\end{array}$ & $\begin{array}{c}\text { Height } \\
(\mathrm{cm})\end{array}$ & $\begin{array}{c}\text { MAS } \\
(\text { grade })\end{array}$ & $\begin{array}{c}\text { MMSE } \\
(\text { score })\end{array}$ & Speech & $\begin{array}{c}\text { Duration } \\
(\text { month })\end{array}$ & $\begin{array}{c}\text { SCP } \\
(\mathrm{score})\end{array}$ \\
\hline 1 & Male & 58 & 65 & 173 & G1+ & 26 & normal & 3 & 5 \\
2 & Female & 65 & 58 & 158 & G1 & 29 & normal & 2 & 5 \\
3 & Male & 62 & 60 & 163 & G1 & 27 & normal & 2 & 5 \\
\hline
\end{tabular}

MAS, Modified Ashworth Scale; MMSE, Mini-Mental Status Examination; SCP, Scale for Contraversive Pushing.

All the procedures were explained to the subjects, and each subject signed an informed consent form.

\section{Design}

This study used a single-subject research design with alternating treatment and multiple baselines across the individuals. In this design, it was possible to have different numbers of baseline sessions among the subjects in order to differentiate from the beginning of the intervention and to compare the effects of the intervention between the experimental conditions in which the intervention was started and stopped. Subjects were separated and performed baseline and intervention sessions, a total of 18 experimental sessions. Subjects 1, 2, and 3 started baseline at the 4th, 7 th, and 10th sessions and intervention at the 14th, 11th, and 8th sessions (Fig. 1). The intervention was applied three times a week for $1 \mathrm{~h}$. All the assessments were performed by a blinded experimenter to remove bias of the measurement variable.

\section{Intervention}

For SPV training with and without visual feedback, an alternating study design was used. The subjects were randomly selected using the sequence of the two training methods upon starting the intervention, and then the subject alternated the training throughout the intervention sessions.

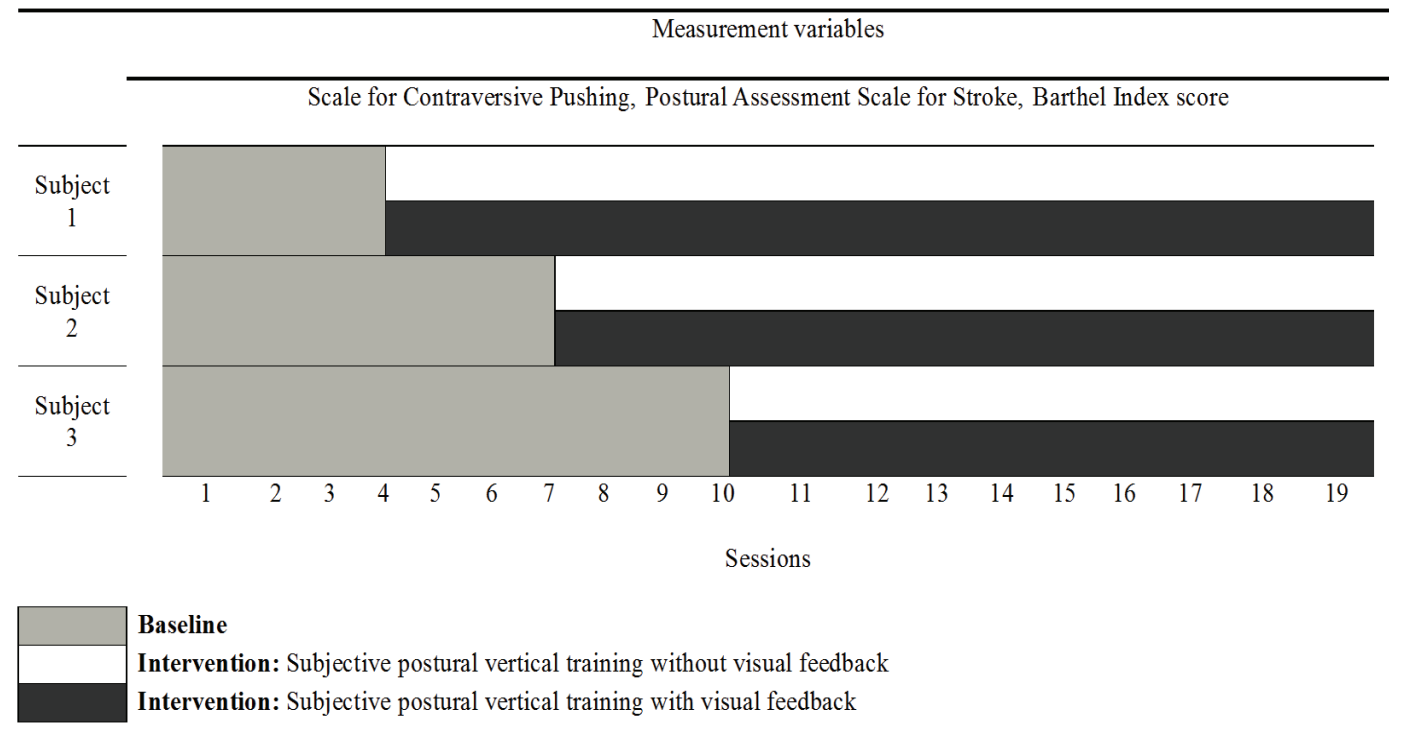

Fig. 1. Experiment process from participant selection to baseline and alternating intervention of subjective postural vertical training with and without visual feedback 
SPV training was performed twice a day, once in the morning and again in the afternoon. Before SPV training without visual feedback was started, the investigator explained the purpose of the intervention to the subject. In the SPV training without visual feedback provided knowledge of performance the investigator read a 5 -min script to the subject regarding how the subject can successfully perform SPV training without visual feedback through self-functional steps. We used the modified SPV training for the functional steps on the basis of the proprioceptive sensory training published by Carey et al. (1993). The modified SPV training was performed in sitting, sit to standing, and standing posture, and they were passively preformed with support and then actively. The subject tried to recognize and find the exact vertical position.

SPV training without visual feedback was performed in a blind situation in a quiet space so that the subjects could concentrate on the task. The process consisted of the following steps. The subject's feet were placed in full contact with the ground on the affected side of the ankle using $2 \mathrm{~kg}$ sandbags; a straight line was made from the nose, chin, and umbilicus, and the shoulder joints were horizontal with the anterior superior iliac spine. The subject's body weight was gradually balanced evenly on both sides, and a waist belt was used to prevent falls. The therapist verbal feedback and auditory feedback on patient were positively used to indicate whether the patient was closer to the correct vertical position; however, if the subject was not near the correct vertical position, feedbacks were not provided. During the initial intervention phase of showing severe pushing behavior, the subject was allowed to receive proper assistance, if necessary.

\section{Outcome measures}

The SCP consists of three distinct items of postural control: 1) symmetry of spontaneous posture while sitting and standing, 2) use of the ipsilesional extremities to abduct and extend the area of physical contact with surfaces such as an arm and hand on a mattress or a leg and foot on the floor while sitting and standing, and 3) resistance to passive correction of posture while sitting and standing (Karnath et al., 2000). The sum total score ranged from 0 (no symptoms) to 6 (severe symptoms). The inter-rater reliability (intraclass correlation coefficient; ICC) is .971, and validity is indicated using the internal consistency measure of Cronbach a, which is 919 (Baccini et al., 2008).

The Postural Assessment Scale for Stroke (PASS) contains 12 four-point items that grade performance in situations of varying difficulty by maintaining or changing a given lying, sitting, or standing posture to measure balance function in stroke patients, including those with very poor performance. Its total score ranges from 0-36. Additionally, its construct validity is .73 , predictive validity is .75 with concomitant Functional Independence Measure, high internal consistency (Cronbach a) is .95, inter-rater reliability is .88 , and test-retest reliability is .72 (Benaim et al., 1999).

The Barthel Index (BI) is a widely used measure of functional disability (Hsueh et al., 2001). Each item score is divided into a 5-point scale: $<24$ points, full dependence; 25-49, dependence; 50-74, partial dependence; 75-90, slight dependence; 91-99, minimal dependence; and 100, full independence. Its inter-rater reliability is .94 , and the internal consistency indicating validity is .92 (Hsueh et al., 2001).

Before the SPV training with and without visual feedback, baseline data on the clinical measures for the SCP, PASS, and BI in subjects 1, 2, and 3 were collected during sessions 4,7 , and 10 to differentiate each subjects' starting time of intervention. All the measures were tested twice, and the average score was used. To determine whether each step of the training was actually followed in sequence by the subject, questions were intermittently asked twice during the SPV training without visual feedback. Since all the subjects answered the questions 
appropriately, it was deemed that they had actively participated in the SPV training without visual feedback. Descriptive statistics and visual analysis methods were used to analyze the results from a graph of measurements at baseline, and intervention data included the typical characteristics of the subjects.

\section{Results}

Subject 1 had right hemiparesis due to hemorrhage in the left anterior cerebral artery and showed slight ankle clonus, hyperactivity of the patellar tendon reflex, no limit in range of motion, fair grade muscle power of the affected side, and good grade muscle power of the unaffected side. He exhibited severe fall down symptoms such as pushing towards the affected side while in the sitting position, and required full assistance to stand. Subject 2 had left hemiparesis due to right middle cerebral artery infarction, with a positive pathological reflex (i.e., the Hoffman sign), hyperactivity of the triceps muscle tendon reflex, a limited range of motion of the elbow joint, poor grade muscle power of the affected side, and good grade of the unaffected side. As for functional level, the subject was able to stand with a lot of assistance. Subject 3 had right hemiparesis due to left middle cerebral artery infarction, with a positive pathological Babinski reflex, general hyperactivity of the deep tendon reflex, a range of motion limitation of the affected side, fair grade muscle power of the affected side, and good grade of the unaffected side. Standing posture was possible with maximal assistance.

He measured SCP and PASS scores and BI at baseline and after therapeutic intervention are presented in Table 2. All the subjects showed an improvement in the BI and SCP and PASS scores after SPV training with and without visual feedback relative to baseline. The BI and the SCP and PASS scores improved after SPV training without visual feedback compared to those after SPV training with visual feedback.

For the SCP score, compared to the average score at baseline, the average score after SPV training without visual feedback decreased from 5.3 to 2.8 in subject 1 , from 4.6

Table 2. Comparison of results of clinical measurements between baseline and intervention

\begin{tabular}{|c|c|c|c|c|}
\hline & \multirow{3}{*}{ Subject } & \multirow[b]{2}{*}{ Baseline } & \multicolumn{2}{|l|}{ Intervention } \\
\hline & & & $\begin{array}{l}\text { SPV training } \\
\text { Without visual feedback }\end{array}$ & $\begin{array}{l}\text { SPV training } \\
\text { with visual feedback }\end{array}$ \\
\hline & & Mean (SD) & Mean (SD) & Mean (SD) \\
\hline \multirow{3}{*}{$\mathrm{SCP}$} & 1 & $5.3(.5)$ & $2.8(1.9)$ & $3.1(1.3)$ \\
\hline & 2 & $4.6(.5)$ & $3.0(1.4)$ & $3.5(1.1)$ \\
\hline & 3 & $3.5(.5)$ & $2.8(1.0)$ & $3.3(.7)$ \\
\hline \multirow{3}{*}{ PASS } & 1 & $14.0(.8)$ & $21.1(4.4)$ & $19.5(2.1)$ \\
\hline & 2 & $15.4(.5)$ & $17.7(2.0)$ & $15.8(.7)$ \\
\hline & 3 & $19.1(.7)$ & $21.7(1.2)$ & $18.4(.5)$ \\
\hline \multirow{3}{*}{ BI } & 1 & $43.8(1.5)$ & $55.0(8.3)$ & $52.0(5.7)$ \\
\hline & 2 & $44.6(1.3)$ & $53.0(6.4)$ & $51.0(3.4)$ \\
\hline & 3 & $51.6(1.6)$ & $60.0(4.7)$ & $60.0(1.9)$ \\
\hline
\end{tabular}

SPV, Subjective Postural Vertical; SCP, Scale for Contraversive Pushing; PASS, Postural Assessment Scale for Stroke; BI, Barthel Index score 


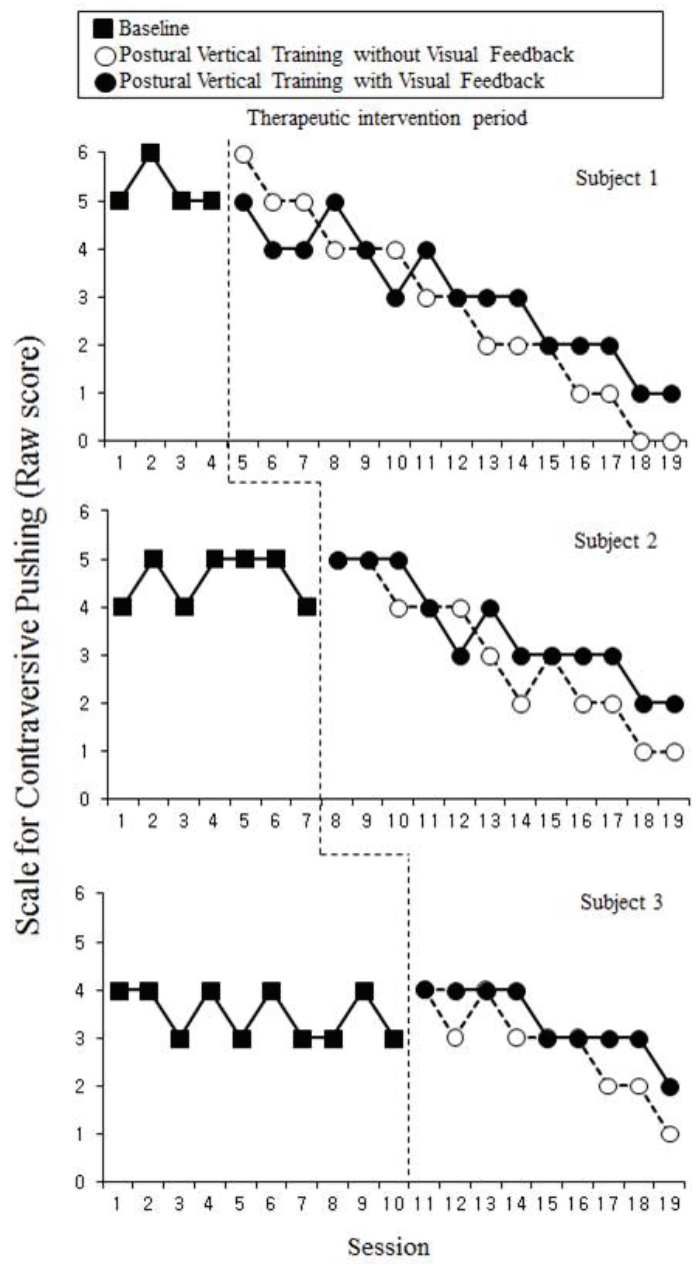

Fig. 2. Changes in Scale for Contraversive Pushing after both interventions

to 3 in subject 2, and from 3.5 to 2.7 in subject 3 (Fig. 2). However, compared to the average score at baseline, the average score after SPV training with visual feedback decreased from 5.3 to 3.1 in subject 1 , from 4.6 to 3.5 in subject 2, and from 3.5 to 3.3 in subject 3 (Fig. 2).

For the PASS score, compared to the average score at baseline, the average score after SPV training without visual feedback increased from 14 to 21.1 in subject 1 , from 15.4 to 17.7 in subject 2, and from 19.1 to 21.7 in subject 3 (Fig. 3). However, compared to the average score at baseline, the average score after SPV training with visual

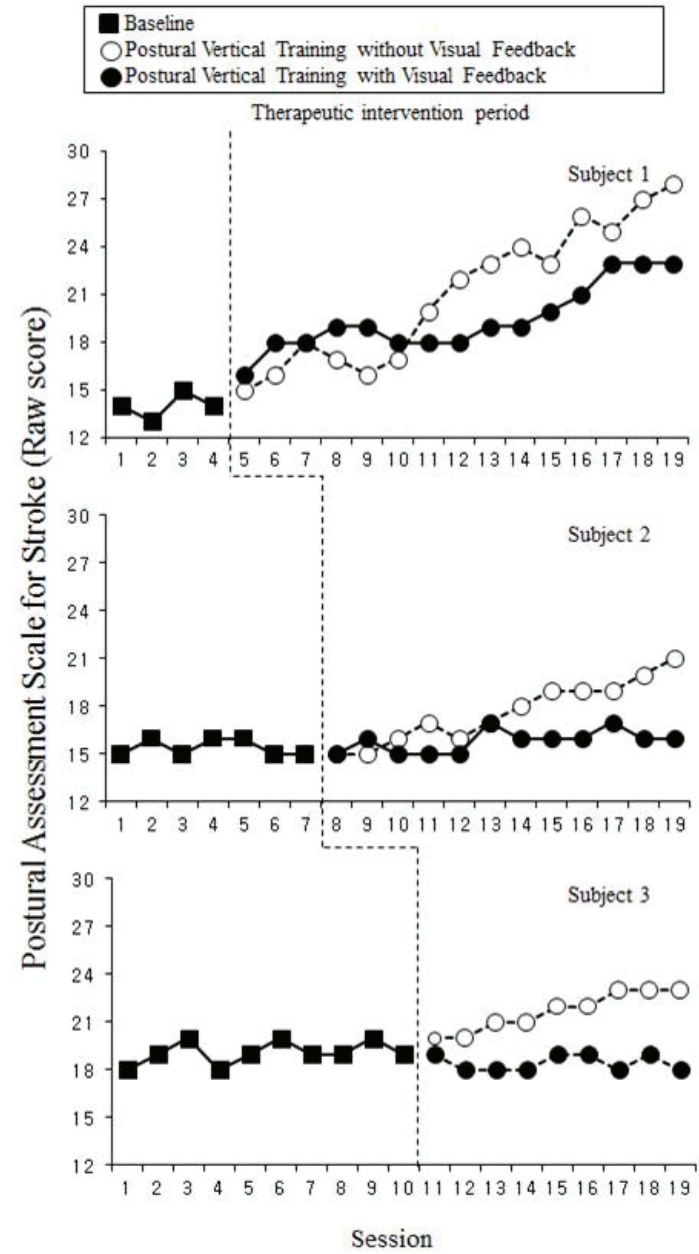

Fig. 3. Changes in Postural Assessment Scale for Stroke after both interventions

feedback increased from 14 to 19.5 in subject 1 , from 15.4 to 15.8 in subject 2, and decreased from 19.1 to 18.4 in subject 3 (Fig. 3).

For the BI, compared to the average BI at baseline, that after SPV training without visual feedback increased from 43.8 to 55 in subject 1, from 44.6 to 52.7 in subject 2, and from 51.6 to 60.4 in subject 3 (Fig. 4). However, compared to the average BI at baseline, that after SPV training with visual feedback increased from 43.8 to 52.3 in subject 1, from 44.6 to 50.5 in subject 2, and from 51.6 to 59.7 in subject 3 (Fig. 4). 


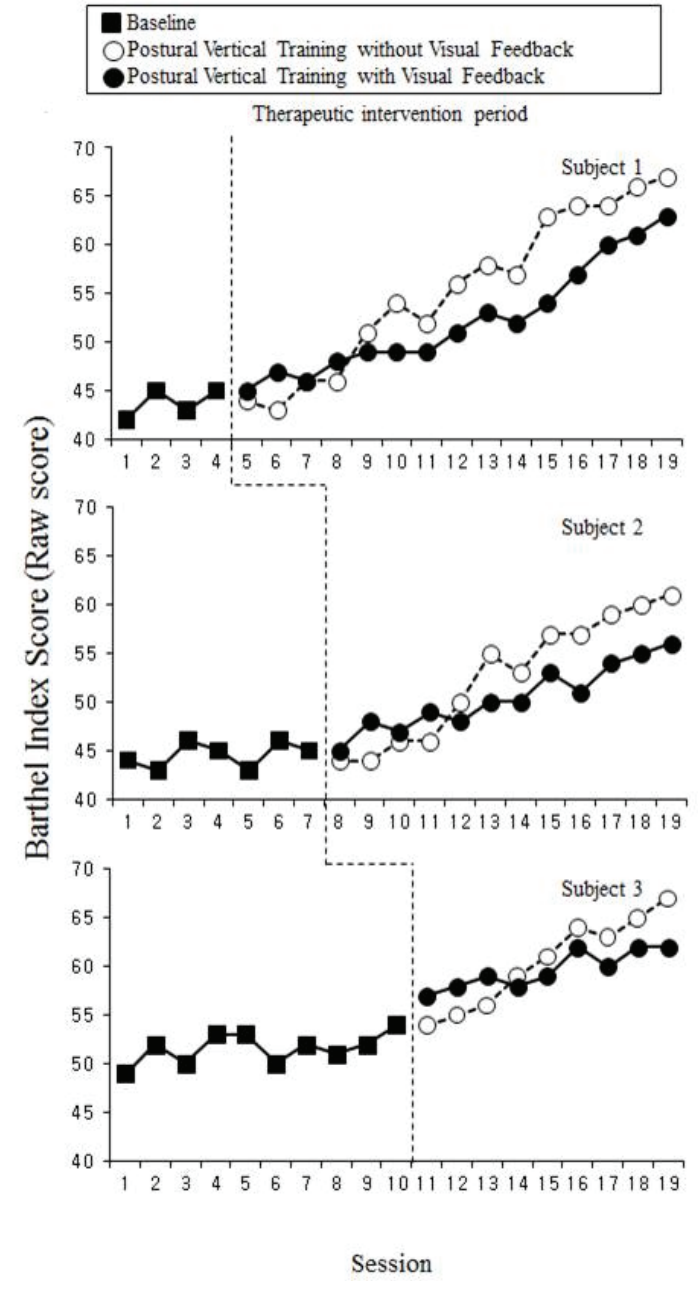

Fig. 4. Changes in Barthel Index score after both interventions

\section{Discussion}

The subjective postural vertical training without visual feedback was more effective than the subjective postural vertical training with visual feedback. Pushing behaviors and functional recovery improved in all three participants when subjective postural vertical training without visual feedback was used. There was a noticeable difference between the two conditions. Therefore, this study finding indicates that the addition of visual feedback is not an effective training method for the time-effective management of pushing behavior and functional activities in stroke patients with pusher syndrome. In addition, intensive proprioceptive training that does not rely on visual feedback has already been reported and is considered to be more effective in the functional recovery of activities of daily living in stroke patients with the pusher syndrome (Karnath and Broetz, 2003; Lee et al., 2015).

Recently, the potential of proprioceptive sensory practice as a method for acquiring and improving motor skills in hemiparetic stroke patients has been recognized in several studies (Sobuh et al., 2014; Wilson et al., 1999; Wong et al., 2012). Neurophysiologically, the proprioceptive sensory factor, which explains the sensory organization in order to balance control, should be incorporated into the visual and vestibular systems (Di Fabio and Badke, 1990). For example, visual disabilities play an important role in the ability of balance control for maintaining a vertical position, and the proprioceptive sensory factor was reported to replace and even be more dominant than the visual sensory factor (Han and Shin, 2013; Horak et al., 1997). Athletic dancers took advantage of the right of the proprioceptive sensory factor (Golomer et al., 2009). Therefore, this can be considered as a rationale for the absence of visual feedback in subjective postural vertical training as a therapeutic strategy for facilitating intensive proprioceptive sensory.

It was reported that it is possible to fully recover from pushing symptoms within 6 months compared to a patient with no pushing symptoms, and patients with pushing behaviors require about 4 week to reach the same functional level (Karnath and Broetz, 2003). This implies that the treatment goal for patients with pusher syndrome is to recover as quickly as possible and to shorten the treatment period. Some researchers (Karnath et al., 2000; Pedersen et al., 1996) argued that visual cues can help the body's vertical orientation to realign, because the orientation perception of the visual system is not impaired by itself. 
Similarly, the use of manual guidance, which reinforces the indication of somesthetic information limiting visual and verbal feedback was reported (Davies, 1985; Pedersen et al., 1996).

To date, most interventions for reducing pushing symptoms have emphasized subjective postural vertical training with visual feedback (Koter et al., 2017; Paci et al., 2009). However, throughout the training in this study, the patients were encouraged to understand the mismatch between the true visual vertical and their own incorrect sense of their body's relation to gravity. The patients learned to move their body mass over the base of support in the context of functional activities; thus, during the absence of visual feedback, attention was paid to the object to be reached and where it was in space rather than to the body movement itself. These responses may be supported by the main points for understanding and effectively treating pusher syndrome, as proposed by Karnath and colleagues (Karnath and Broetz, 2003).

The Scale for Contraversive Pushing, modified Scale for Contraversive Pushing, and Burke Lateropulsion Scale used to assess the pushing behavior symptoms in post-stroke patients have a high reliability and validity. However, we used the Scale for Contraversive Pushing, because it is the most popular examination tool that is quick and simple to use with the highest reliability and validity (Baccini et al., 2008; Koter et al., 2017). The Postural Assessment Scale for Stroke was used, because it is one of the most valid and reliable clinical assessments of postural control in stroke patients during the first 6 months after stroke (Baccini et al., 2008). The Barthel Index was selected in this study because it is the most commonly used measurement for assessing the functional capacity in the activities of daily living at different positions in stroke patients (Hsueh et al., 2001).

The subjective postural vertical training with visual feedback was more effective than subjective postural vertical training without visual feedback in the early intervention stages according to the Scale for Contraversive Pushing score; however, subjective postural vertical training without visual feedback was more effective according to a longer intervention stage. Subject 1 performed subjective postural vertical training with visual feedback and improved by up to $41.6 \%$ during 6 weeks of interventions, while during subjective postural vertical training without visual feedback the improvement was up to $46.7 \%$. This suggests that the improvement was very effective, considering that this study occurred over a short time.

For the Postural Assessment Scale for Stroke score, the average score of the subjective postural vertical training without visual feedback increased by up to $51 \%$, while the score of the subjective postural vertical training with visual feedback increased by up to $39 \%$. Most studies report that $80 \%$ of pusher syndrome patients have proprioceptive sensory problems (Carey et al., 1993; Lee et al., 2013) located in the muscle, joint, and posture causing imbalance; thus, solving proprioceptive problems in various positions is considered to be improved postural alignments in these patients. The intensive proprioceptive training should play the most important role in solving the problems of abnormal sensory and motor abilities in stroke patients (Chae and Lee, 2010; Wong et al., 2012).

For the Barthel Index score, subjective postural vertical training with visual feedback was more effective than subjective postural vertical training without visual feedback during the early intervention stages; similarly, subjective postural vertical training without visual feedback was more effective during the longer intervention stage. For the Barthel Index score, subjective postural vertical training without visual feedback was improved by up to $26.6 \%$, while subjective postural vertical training with visual feedback was improved by up to $17.1 \%$. According to the Barthel Index score, it took 19 week to reach full independence ( $\geq 95$ points) in patients with pusher syndrome, while it took 13 week in patients without the 
pusher syndrome (Pedersen et al., 1996). In this study, subjective postural vertical training without visual feedback at 6 week in subject 1 showed about 11 points in improvement. This improvement rate is considered to be caused by a combination of the two alternating training methods used, which may have a positive effect on the overall activities of daily living as the pushing symptoms were reduced.

Nevertheless, this study had several limitations in interpreting and verifying the results of this intervention. First, this individual single subject design was too small to generalize the results to all pusher syndrome patients. Second, in alternating the two intervention approaches of subjective postural vertical training with and without visual feedback, the carry-over effect from one to another was not firmly controlled. Third, regarding the duration of the intervention's effect, the long-term follow-up effects of the intervention couldn't clarify because this design requires evaluating only the initial effect to the intervention from the intervention. Therefore, future controlled studies with larger sample sizes and longer interventions are required to determine the clinical benefits of the intensive proprioceptive sensory inputs without visual feedback as a subjective postural vertical training in stroke patients with pusher syndrome.

\section{Conclusion}

This present study suggests that SPV training without visual feedback is beneficial for the time-effective management of abnormal pushing behavior and is associated with functional activities of stroke patients with the pusher syndrome. However, caution should be exercised in interpreting these results and when implementing future studies, because these results cannot be generalized to all stroke patients with the pusher syndrome.
Acknowledgements

This research was supported by Basic Science Research Program through the National Research Foundation of Korea (NRF) funded by the Ministry of Education (2017R1D1A3B03031876).

\section{References}

Baccini M, Paci M, Nannetti L, et al. Scale for contraversive pushing: cutoff scores for diagnosing "pusher behavior" and construct validity. Phys Ther. 2008;88(8):947-55.

Benaim C, Perennou DA, Villy J, et al. Validation of a standardized assessment of postural control in stroke patients: the Postural Assessment Scale for Stroke Patients (PASS). Stroke. 1999;30(9):1862-8.

Bonan IV, Hubeaux K, Gellez-Leman MC, et al. Influence of subjective visual vertical misperception on balance recovery after stroke. J Neurol Neurosurg Psychiatry. 2007;78(1):49-55.

Broetz D, Johannsen L, Karnath HO. Time course of 'pusher syndrome' under visual feedback treatment. Physiother Res Int. 2004;9(3):138-43.

Carey LM, Matyas TA, Oke LE. Sensory loss in stroke patients: effective training of tactile and proprioceptive discrimination. Arch Phys Med Rehabil. 1993;74(6): 602-11.

Chae JB, Lee MH. The effects of somatosensory training on the spatiotemporal gait parameters and balance in patients with stroke. J Korean Soc Phys Med 2010;5(4):587-96

Davies PM. Steps To Follow: A Guide to the Treatment of Adult Hemiplegia. Berlin. Springer-Verlag. 1985.

Di Fabio RP, Badke MB. Relationship of sensory organization to balance function in patients with hemiplegia. Phys Ther. 1990;70(9):542-8. 
Golomer EM, Gravenhorst RM, Toussaint Y. Influence of vision and motor imagery styles on equilibrium control during whole-body rotations. Somatosens Mot Res. 2009;26(4):105-10.

Han KB, Shin WS. Effects of trunk position sense through visual cue deprivation balance training in subacute stroke. J Korean Soc Phys Med. 2013;8(3):327-35.

Horak FB, Henry SM, Shumway-Cook A. Postural perturbations: new insights for treatment of balance disorders. Phys Ther. 1997;77(5):517-33.

Hsueh IP, Lee MM, Hsieh CL. Psychometric characteristics of the Barthel activities of daily living index in stroke patients. J Formos Med Assoc. 2001;100(8):526-32.

Karnath HO, Broetz D. Understanding and treating "pusher syndrome". Phys Ther. 2003;83(12):1119-25.

Karnath HO, Ferber S, Dichgans J. The origin of contraversive pushing: evidence for a second graviceptive system in humans. Neurology. 2000;55(9):1298-304.

Koter R, Regan S, Clark C, et al. Clinical Outcome Measures for Lateropulsion Poststroke: An Updated Systematic Review. J Neurol Phys Ther. 2017;41(3):145-55.

Lee DH, Choi SJ, Choi HS, et al. Comparison of visual and auditory biofeedback during sit-to-stand training for performance and balance in chronic stroke patients. J Korean Soc Phys Med. 2015;10(4):59-68.

Lee JH, Kim SB, Lee KW, et al. Somatosensory findings of pusher syndrome in stroke patients. Ann Rehabil
Med. 2013;37(1):88-95.

Paci M, Baccini M, Rinaldi LA. Pusher behaviour: a critical review of controversial issues. Disabil Rehabil. 2009;31(4):249-58.

Pedersen PM, Wandel A, Jorgensen HS, et al. Ipsilateral pushing in stroke: incidence, relation to neuropsychological symptoms, and impact on rehabilitation. The Copenhagen Stroke Study. Arch Phys Med Rehabil. 1996;77(1):25-8.

Priplata AA, Patritti BL, Niemi JB, et al. Noise-enhanced balance control in patients with diabetes and patients with stroke. Ann Neurol. 2006;59(1):4-12.

Shumway-Cook A, Woollacott MH. Motor control: Theory and practical application (1sted). Baltimore. Lippincott Williams \& Wilkins. 1995.

Sobuh MM, Kenney LP, Galpin AJ, et al. Visuomotor behaviours when using a myoelectric prosthesis. J Neuroeng Rehabil. 2014;11:72.

Stevens JA, Stoykov ME. Using motor imagery in the rehabilitation of hemiparesis. Arch Phys Med Rehabil. 2003;84(7):1090-2.

Wilson LR, Gandevia SC, Inglis JT, et al. Muscle spindle activity in the affected upper limb after a unilateral stroke. Brain. 1999;122(11):2079-88.

Wong JD, Kistemaker DA, Chin A, et al. Can proprioceptive training improve motor learning? J Neurophysiol. 2012;108(12):3313-21. 